\title{
Bandwidth and Gain improvement by using suspended Fractal MSA at 2.4GHZ
}

\author{
Prajakta B.Jadhav ${ }^{1}$, Prof.Mrs.M.M.Pawar ${ }^{2}$ \\ ${ }^{I}$ (SVERI college of Engineering, Pandharpur, India) \\ ${ }^{2}$ (SVERI college of Engineering, Pandharpur, India)
}

\begin{abstract}
The paper present design of microstrip patch antenna at $2.4 \mathrm{GHz}$. Further by introducing fractal concept to the star-shaped microstrip antenna Koch curve antenna is designed. The Koch island fractal patch antenna is introduced in order to reduce the antenna size. By space-filling property of fractal geometry, this antenna reveals lower resonant frequency. Based on experimental result, it is found that as iteration and iteration factor increases, the resonant frequency of this patch antenna decreases. Broad band operation with size reduction is obtained. To improve efficiency and bandwidth suspended configuration is used. A comparison of fractal antenna with conventional microstrip patch antenna is made regarding the bandwidth, return loss, VSWR, and gain. The antenna is simulated by the HFSS software.(High frequency structure simulator).
\end{abstract}

Keywords: Bandwidth enhancement, Fractal geometry, HFSS, Microstrip patch antenna, Koch curve.

\section{Introduction}

The increasing range of wireless telecommunication services and related applications is driving the attention to the design of multifrequency (multiservice) and small antennas. Operators are looking for systems that can perform over several frequency bands or are reconfigurable as the demands on the system changes. Some applications require the antenna to be as miniaturized as possible as can be done [1]. Antenna is a transducer designed to transmit or receive electromagnetic waves. Microstrip antennas have several advantages over conventional microwave antenna and therefore are widely used in many practical applications [3].Microstrip antennas have low profile, low cost, light weight and conveniently to be integrated with RF devices. The size of the micros trip antennas becomes too large to be manageable. Reduction of antenna size becomes extremely important in wireless communications and hence it is desired to bring down the size of antenna while achieving the same performance of the large size antenna. Fractal geometry plays a prominent role for these requirements. Fractals have non-integral dimensions and their space filling capability could be used for miniaturizing antenna size. To overcome microstrip antenna's limitation of narrow bandwidth by generating more than one resonant frequency many techniques have been used in the past e.g. different shaped slots, multilayer, stack, two folded parts to the main radiating patch and use of air gap have been proposed and investigated. In the design presented in this paper use of air gap i.e. Suspended technique is used.

\section{Fractal Geometry Antenna}

Fractal was first defined by Benoit Mandelbrot in 1975 as a way of classifying structures whose dimensions were not whole numbers.[4] A fractal is a rough or fragmented geometric shape that can be subdivided in parts, each of which is (at least approximately) a reduced-size copy of the whole. Fractals are generally self-similar and independent of scale. There are many mathematical structures that are fractals; e.g. Sierpinski's gasket, Cantor's comb, von Koch's snowflake Fractals also describe many real-world objects, such as clouds, mountains, turbulence, and coastlines that do not correspond to simple geometric shapes. Fractal geometry is a very good solution to reduce the size of antenna. Fractal shaped antennas show some interesting features which results from their geometrical properties. The unique features of fractals such as self-similarity and space filling properties enable the realization of antennas with interesting characteristics such as multi-band operation and miniaturization. In this paper we used Koch's snowflake fractal antenna

\subsection{Properties of Koch antenna}

The Koch curve has an infinite length because each iteration creates four times as many line segments as in the previous iteration, with the length of each one being one-third the length of the segments in the previous stage. Hence the total length of the curve increases by one third with each iteration and the length of the curve after $\mathrm{n}$ iterations will be ${ }^{\mathrm{n}}(4 / 3)$ times the original triangle perimeter, which is unbounded as $\mathrm{n}$ tends to infinity. The fractal dimension of the Koch curve is $\log 4 / \log 3 \approx 1.26186$. This is greater than the dimension of a line but less than Peano's curve. The Koch curve is continuous everywhere but differentiable nowhere. Different iteration stages of triangular Koch curve is shown in figure 1. 


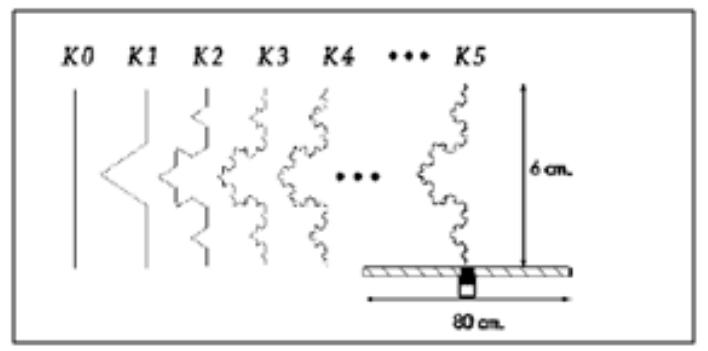

Fig.1different itration stages of triangular Koch curve

\section{Antenna Design}

In this paper to design the Suspended Koch antenna HFSS (high frequency structure simulator) software is used. The Koch snowflake can be constructed by starting with an equilateral triangle, then recursively altering each line segment as follows:

1. Divide the line segment into three segments of equal length.

2. Draw an equilateral triangle that has the middle segment from step 1 as its base and points outward.

3. Remove the line segment that is the base of the triangle from step 2.[6]

After one iteration of this process, the resulting shape is the outline of a hexagram. The Koch snowflake is the limit approached as the above steps are followed over and over again. The Koch curve originally described by Koch is constructed with only one of the three sides of the original triangle. In other words, three Koch curves make a Koch snowflake. First 3 iteration of Koch curve is shown in fig 2(a), (b), (c).

There are three essential parameters for design of patch antenna i.e. resonant frequency (fr), dielectric material of the substrate (Er), and the thickness of substrate (D). Here $2.45 \mathrm{GHz}$ has been chosen as the resonant frequency. The dielectric material of the substrate selected for this design is FR-4 which has a dielectric constant (Er) of 4.4

The fundamental mode resonant frequency of such antenna is given by

Where,

$$
\mathrm{Fr}=\frac{2 \mathrm{C}}{3 \mathrm{~W} \sqrt{\mathrm{Er}}}
$$

$$
\begin{aligned}
& \mathrm{C}=\text { speed of light } \\
& \mathrm{Er}=\text { relative permittivity of substrate } \\
& \text { Then patch side length } \mathrm{W} \text { is given by }
\end{aligned}
$$

$$
\begin{gathered}
\mathbf{W}=\frac{2 \mathrm{C}}{3 \mathbf{f r} \sqrt{\mathrm{Er}}} \\
\text { Lambda }=\frac{\mathbf{C}}{\mathbf{f}} \\
\mathrm{F}=2.4 \mathrm{Ghz} \\
\mathrm{Er}=4.4 \\
\text { Ground Length }=\text { lambda/2 }
\end{gathered}
$$

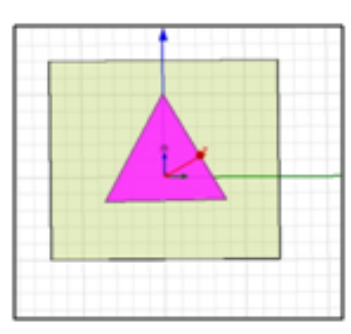

(a)

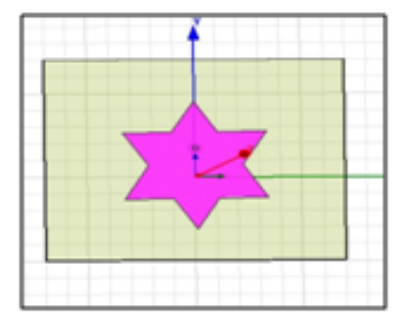

(b)

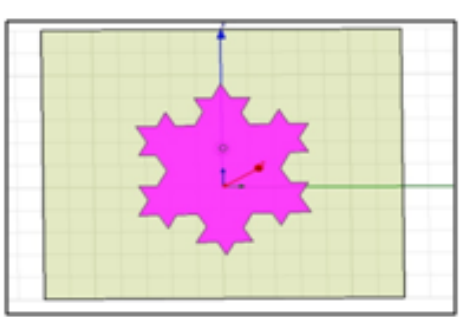

(c)

Fig.2 (a), (b) and (c) illustrates the simple MSA, Koch curve Iteration-1 and Koch curve Iteration-2

In order to improve bandwidth air gap is introduced means suspended technique is used. The configuration yielded improved measured bandwidth with dual frequency band. Suspended configuration is shown in fig 3. 


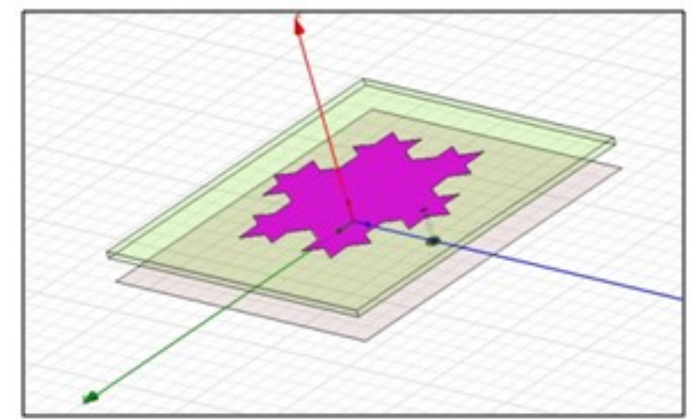

Fig. 3 Suspended Fractal antenna

\section{Simulation \& Results Discussion}

The figure. 3 shows the HFSS generated design of Suspended Koch curve antenna. The VSWR, return loss, gain are computed using HFSS. As per the theory voltage standing wave ratio should be $\leq 2$.Idealy it should be1.The simulated VSWR for simple MSA, VSWR for Koch curve Iteration-1and Iteration 2 and VSWR for Suspended Fractal antenna is as shown in fig.4. (a), (b), (c) and (d) respectively. The VSWR, which can be derived from the level of reflected and forward waves.

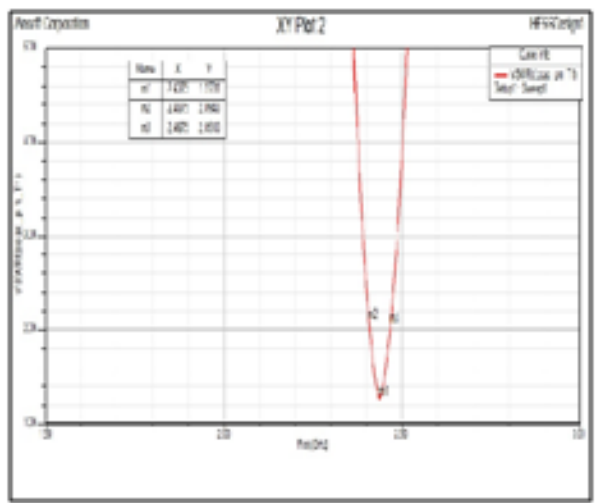

(a)

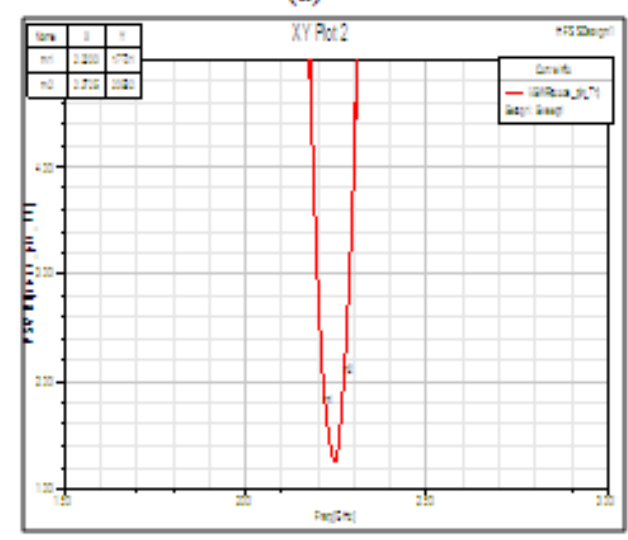

(c)

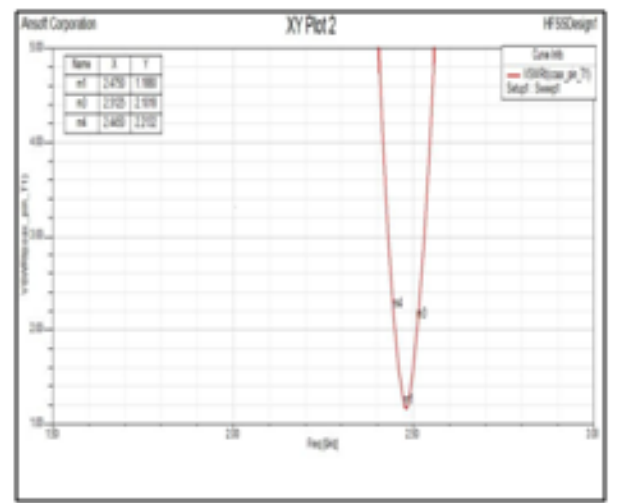

(b)

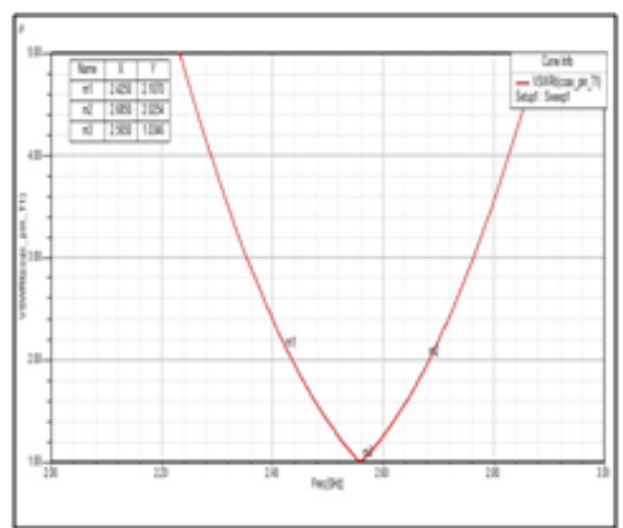

(d)

Fig. 4(a) VSWR for simple MSA, (b) VSWR forKoch curve Iteration-1, (c) VSWR for Koch curve Iteration2 (d) VSWR for Suspended Fractal antenna

Return loss indicates the amount of power that is lost to load and does not return as reflection. The simulated return loss for simple MSA, Koch curve Iteration-1and Iteration 2 and Suspended Fractal antenna shown in fig.5 (a), (b), (c) and (d) respectively 


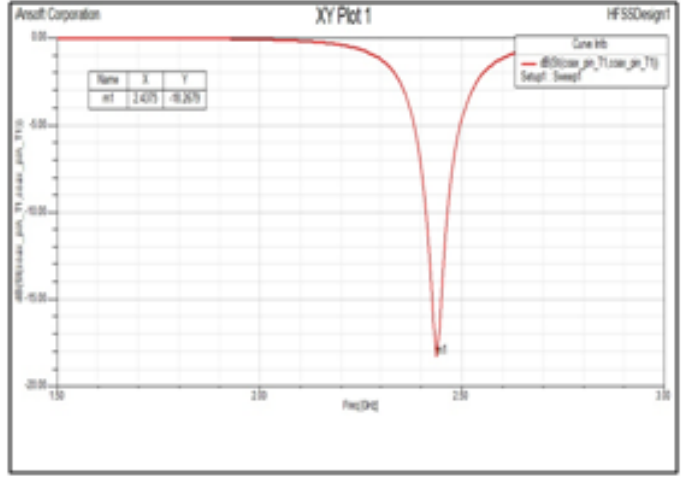

(a)

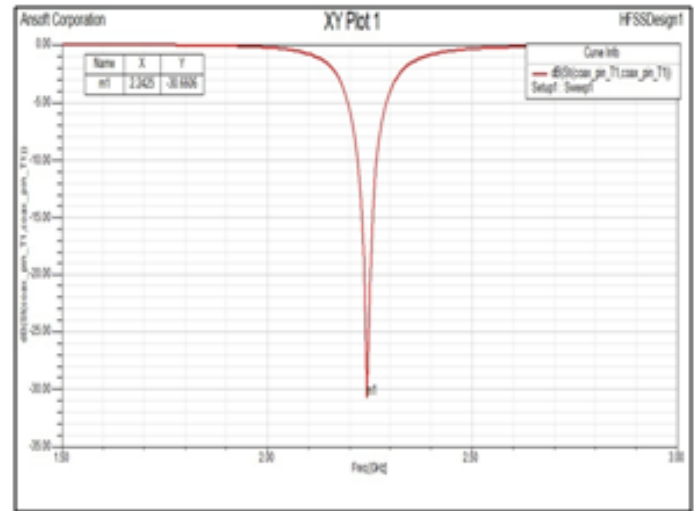

(c)

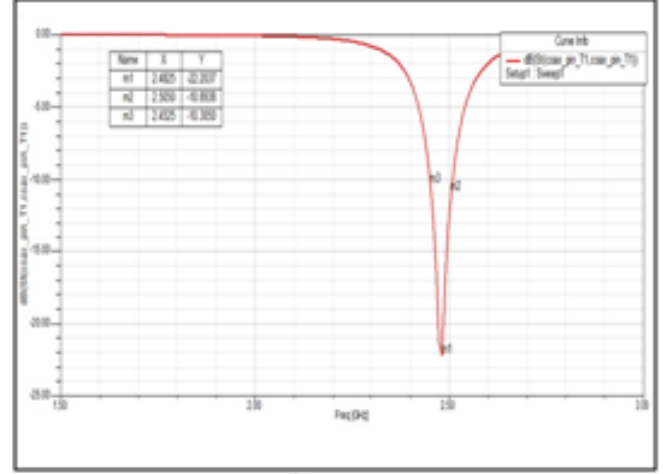

(b)

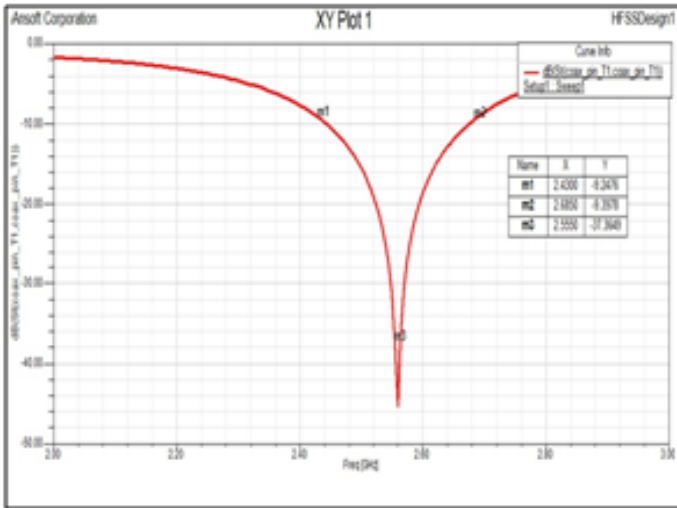

(d)

Fig. 5(a) Return loss for simple MSA, (b) Return loss for Koch curve Iteration-1, (c) Return loss for Koch curve Iteration2 (d) Return loss for Suspended Fractal antenna

The gain of an antenna is essentially a measure of the antenna's overall efficiency. The simulated gain for simple MSA, Koch curve Iteration-1and Iteration 2 and Suspended Fractal antenna shown in fig.6 (a), (b), (c) and (d) respectively

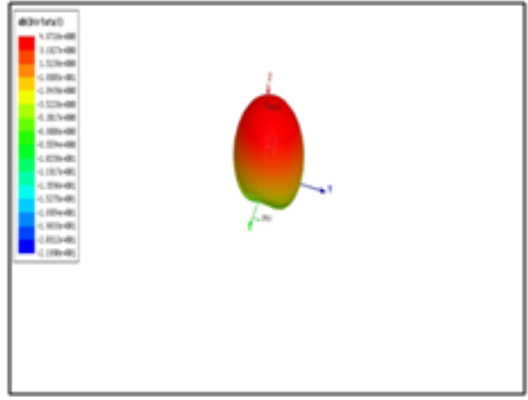

(a)

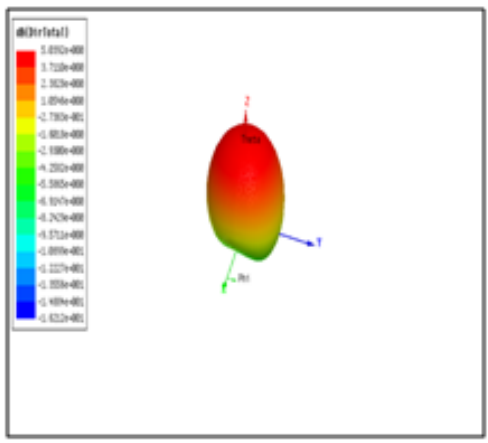

(c)

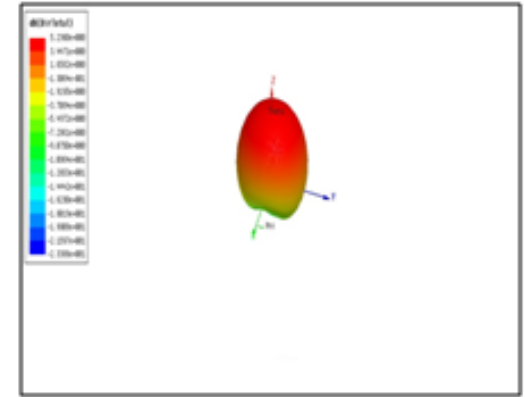

(b)

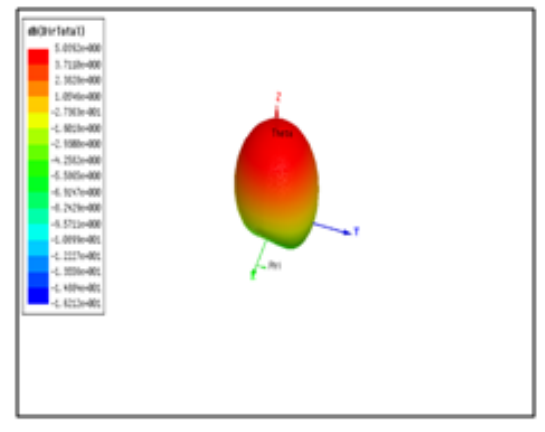

(d)

Fig. 6(a) gain for simple MSA, (b) gain for Koch curve Iteration-1, (c) gain for Koch curve Iteration2 (d) gain for Suspended Fractal antenna 
The Table1 gives all the summarized results of the four proposed antennas

Table.1comparative Result For Triangular Microstrip Antenna, Star Microstrip Antenna, Koch Curve Antenna, Suspended Fractal Antenna

\begin{tabular}{|c|c|c|c|c|c|c|}
\hline $\begin{array}{c}\text { Sr. } \\
\text { No. }\end{array}$ & $\begin{array}{c}\text { Types of Microstrip } \\
\text { antenna }\end{array}$ & $\begin{array}{c}\text { Frequency } \\
(\mathrm{GHz})\end{array}$ & $\begin{array}{c}\text { Return } \\
\text { loss(dB) }\end{array}$ & VSWR & $\begin{array}{c}\text { B.W } \\
(\mathrm{MHz})\end{array}$ & Gain (dB) \\
\hline 1) & $\begin{array}{c}\text { Triangular } \\
\text { Microstrip antenna }\end{array}$ & 2.43 & -18.26 & 1.27 & 52 & 4.87 \\
\hline 2$)$ & $\begin{array}{c}\text { Star Microstrip } \\
\text { antenna1: iteration }\end{array}$ & 2.48 & -22.21 & 1.18 & 52 & 5.23 \\
\hline 3$)$ & Koch 2 2 in iteration & 2.24 & -18.57 & 1.77 & 42 & 5.0392 \\
\hline \multirow{2}{*}{$4)$} & $\begin{array}{c}\text { final suspended } \\
\text { fractal antenna }\end{array}$ & $1) 2.55$ & -37.36 & 2.1 & 260 & 5.92 \\
\cline { 3 - 7 } & $2) 4.14$ & -16.17 & 1.37 & 290 & 7.31 \\
\hline
\end{tabular}

From above table it is clear that when iteration increases, the resonant frequency of this patch antenna shifted to lower side i.e. decreased and after each iteration gain is increased. And we get dual frequency band with large bandwidth.

\section{Conclusion}

The resonance behavior and space filling capabilities of the Koch curve fractal antenna have been investigated. It is found that as the resonant frequencies decreased and after each iteration gain is increased. As the generating iteration is increased by introducing number of triangle and length of one antenna was reduced by bending the $2 / 3$ pattern of the Koch fractal antenna counter clockwise. Then, again rotating whole antenna $90^{\circ}$ counter clockwise with specific dimension improves the antenna gain as well as its bandwidth. The proposed antenna have some favorable characteristics such as; compact size, almost symmetrical radiation pattern, higher gain, satisfactory return loss less than $10 \mathrm{db}$ and acceptable bandwidth in desired frequency (2.4$2.483 \mathrm{GHz}$ )ISM(Industrial scientific medical)band. Due to suspended configuration we get dual frequency band with large bandwidth.

\section{References}

[1]. Divya Dixit Saxena and Ankur Kumar Tripathi "Fractal based microstrip patch antenna in wireless communication".

[2]. Ms.Priyanka, Mr. Navin Srivastava, "Bandwidth enhancement for microstrip patch antenna using suspended techniques for wireless applications "International Journal of Advancements in Research \& Technology,Volume2, Issue5, May-2013.

[3]. Indrasen Singh, Dr. V.S. Tripathi "Micro strip Patch Antenna and its Applications: a Survey".

[4]. Shubhangi S. Ghorpade Vidya B. Babare V. U. Deshmukh "Comparison Of E-Shape Microstrip Antenna And E-Shape Fractal Antenna".

[5]. M. Ramkumar Prabhu, V. Reji and 3A. Sivabalan "Improved Radiation and Bandwidth of Triangular and Star Patch Antenna" Research Journal of Applied Sciences, Engineering and Technology 4(12): 1740-1748, 2012

[6]. Dr. K.J.Vinoy "Size reduction of low frequency micro strip patch antennas with Koch fractal slots".

[7]. Homayoon Oraizi and Shahram Hedayati Combined Fractal Geometries for the Design of Wide Band Microstrip Antennas with Circular Polarization PIERS Proceedings, Suzhou, China, September 2011.

[8]. C.L.Mac,R.Chair,K.F.Lee,K.M.LukandA.A.Kishk “ half U-slot patch antenna with shorting wall”, electronic letters vol 39,pp 1779$1780,2009$.

[9]. Balanis, C. A “Antenna Theory” John Wiley \& Sons, Inc., New York, 2004.

[10]. C.L.Mac,R.Chair,K.F.Lee,K.M.LukandA.A.Kishk”half U-slot patch antenna with shorting wall”, electronic letters vol 39,pp 17791780,2009 